\title{
Chasing Mutants
}

\author{
Adam Leon Smith
}

\begin{abstract}
This chapter describes mutation testing, how it has developed, the types of tools associated with it, the benefits to a quality assurance process, and the associated challenges with scaling it as a test design technique and as a consumer of resources.
\end{abstract}

Keywords Software testing · Software quality · Test automation · Mutation testing

\section{Introduction}

It's hard to prove a negative, and if you are reading this book, you are likely to be aware that testing proves the presence of defects, rather than the absence. Mutation testing turns this principle on its head and asks, if we know there are defects, what do our test results tell us about the quality of our software and tests?

As engineers increasingly turn to more automated software verification approaches and higher quality software outputs are demanded by ever-reducing release timelines, mutation testing helps us take a step back to assess whether we should really be so confident in our tests.

I asked Markus Schirp, author of Mutant [1], a mutation testing tool for Ruby, how he defined mutation testing:

Mutation testing is the process of heuristically determining semantics of your program that are not covered by tests.-Markus Schirp

With most software testing approaches, it is hard to determine if you can detect failures in the testing process until the failures occur in a later testing activity, or

\footnotetext{
A. L. Smith

Dragonfly, London, UK

(C) The Author(s) 2020

S. Goericke (ed.), The Future of Software Quality Assurance, https://doi.org/10.1007/978-3-030-29509-7_12
} 
worse, in a production environment. This is something familiar to every test manager as they deal with the learning from production releases and feeding information back into the test process. Surely there is a better way to find problems with test coverage?

Mutation Testing is a testing technique that can be traced back to 1971 [2], which has gained more and more attention, now with over a dozen associated tools and used in a range of software contexts. The number of tools available has grown significantly from less than 5 in 1981, to over 40 in 2013 [3].

Mutation Testing sits in a gray area of testing techniques that don't rely on formal specification of the desired behavior of the system, alongside fuzz testing, metamorphic testing, and arguably exploratory testing.

At its core, mutation testing is the practice of executing a test, or a set of tests, over many versions of the software under test-a so-called mutant. Each version of the software under test has different faults deliberately and programmatically injected. Each testing iteration is conducted on a slightly different version of the software under test, with different faults injected based on heuristics, or "rules of thumb" that correspond to common faults. These versions are referred to as mutants, in the context that they are small variations. The purpose of the testing is usually to determine which faults are detected by test procedures, resulting in failures.

The manufactured faulty versions of the software aren't called mutants because they are inferior, but because they are changed in the same way that human genetics mutate as part of natural evolution. This is similar to the use of genetic algorithms in artificial intelligence to solve search and optimization problems.

The benefits of mutation testing can be significant, it can give enormously useful insight into:

- The quality and coverage of automated tests, in particular, coverage of assertions and validations

- The coverage of testing in a particular area of the software

- The success of particular test design techniques

- The complexity and maintainability of different areas of code

- The traceability of software code or components to the overall business functionality, through the tests

It can be used as an approach to improve an existing set of tests, or as a tool to help build a new test suite, either to ensure it has sufficient validations, or to prioritize testing towards areas of the code. Ultimately, it provides a set of facts about quality, information that is not otherwise available (Fig. 1).

\section{Automated Testing}

Mutation Testing conceptually is not limited to automated tests; in principle it is an approach that could be applied to manual testing. However, the cost associated with running thousands of tests manually (on code that will never reach production, in order to improve the development and testing process) doesn't stack up against the benefit in most cases. 


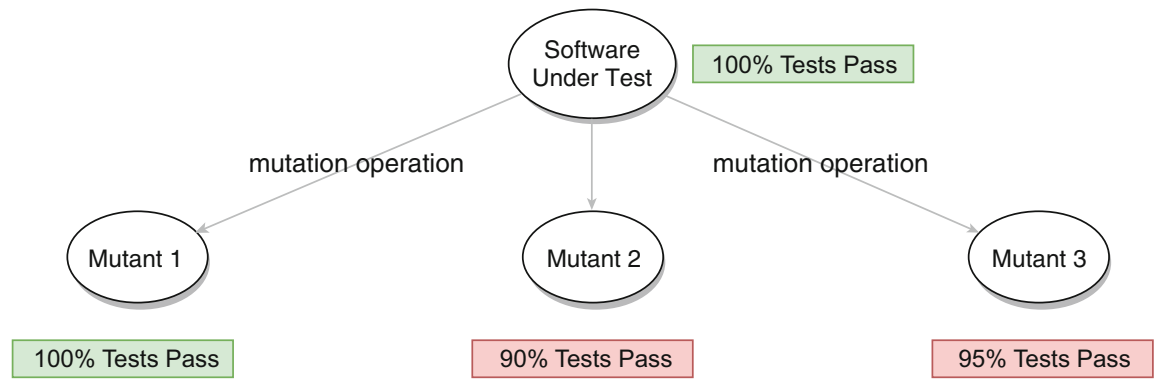

Fig. 1 In this example, Mutant 1 doesn't cause any tests to pass, indicating that the injected faults is not detected, hence there is a fault in the test coverage. Mutants 2 and 3 cause test failures, which is good!

One of the core challenges with automated testing is ensuring that there is the right balance of assertions and validation checks to ensure that software is working, without making the test suite brittle to irrelevant structural changes in the software. Although usually slower, we know that human testers find more defects in software. We know that the reason for this is that the human tester has more context, and uses experience and heuristics to determine correct behavior as well as the stated assertions in any testing procedure. Unlike with manual testing, it is a given that automated test suites are by nature, only going to detect defects within the bounds of the validations they have been setup to perform-in other words, they will only check the things they have been told to check.

In the most extreme example, it is possible to create an automated test that starts the software under test and validates it appears to execute successfully, without making any assertions about the program output. This would be a very maintainable test, as it would rarely need to be updated to stay in line with the software, but a very low value test, as it tells us little other than that the program executes. If this hypothetical software and test were to be evaluated using mutation testing, the score would be very low, as mutated versions of the software would only fail the testing if the injected fault caused a full execution failure.

Taking a counter-example, an automated test against a website that compares every single accessible element of the HTTP responses against a baseline copy. This would be a very unstable test, as it would fail at even the smallest change, perhaps one that a real user or tester could not perceive. If this software and test were evaluated with mutation testing, the score would be extremely high, as virtually any change would cause a test failure.

Mutation testing does not solve the problem of the maintainability of test automation, but it does give useful insight into the value actually offered by individual tests.

This is also important because even in an environment with a high level of automation, exhaustive or full combinatorial testing is often not performed because it is considered an impractical use of resources.

One risk with any test design technique is test case explosion-that is, when you focus on a specific test design technique, and the volume of tests dramatically 
increases. This might be because you are just starting to apply format test design techniques, or it might be because you are focusing too much on one technique.

Mutation testing information can help you prioritize resources to maximize test coverage in new ways.

\section{Code Coverage and Mutation Operators}

On the one hand, mutation testing is a black-box testing technique, as the design and execution of the tests do not require knowledge of the inner workings of the code. That said, the mutation operators and the fault injection process must have very detailed knowledge of the code, or at least, a mutable derivation of it. In fact, the set of mutants generated is inextricably linked to the language and structure of the underlying code, but then again so are real faults.

When the mutation "operator" injects faults into the code, it performs rule-based changes, which can usually be customized by the engineers working on the code. Some example of these include:

- Deleting statements from the code

- Inserting statements from code

- Altering conditions in code

- Replacing variable values

Let's go over some basic programming concepts, and how they link to faults, code coverage and mutation testing. In most programming languages, an executable statement expresses an action to be carried out, such as assigning a value, e.g. true or false, to a variable. The ISTQB glossary defines this as: "A statement which, when compiled, is translated into object code, and which will be executed procedurally when the program is running and may perform an action on data."

For example, in the code below, the statements all of the lines that don't start IF/ELSE are statements:

\section{Pseudo Code}

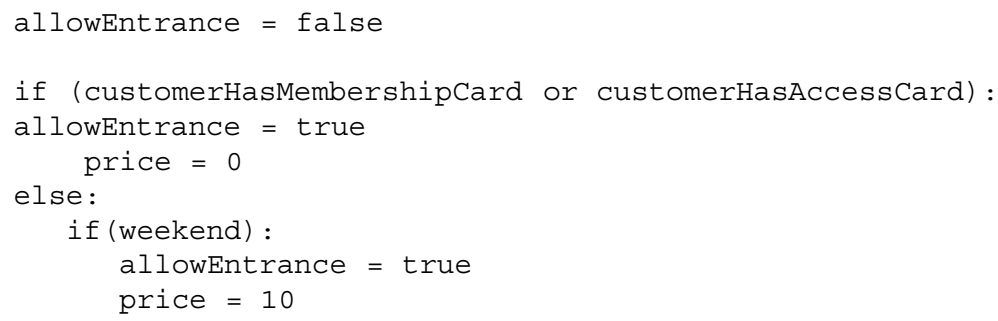


The degree to which statements are covered by an executable test suite is usually described as statement coverage. Statement coverage is the percentage of executable statements that have been exercised by a test suite.

$$
\text { Statement Coverage }=\frac{\text { Number of Executed Statements }}{\text { Total Statements }}
$$

To reach full statement coverage on the code above you would need two tests, as there are two exclusive paths through the code required to execute each statement.

Another coverage approach is to cover each branch or decision, essentially wherever a conditional statement such as if, for, while is included, ensure both outcomes of the conditional statement are evaluated. To reach full branch coverage on the example above, you would need one further test, which covered whether the weekend variable was true or false.

$$
\text { Branch Coverage }=\frac{\text { Number of Executed Branches }}{\text { Total Branches }}
$$

Finally, condition coverage, is a code coverage metric which measures whether each individual condition has been evaluated to true or false. This can be calculated as:

$$
\text { Condition Coverage }=\frac{\text { Number of Executed Operands }}{\text { Total Operands }}
$$

So referring again to the example above, ensuring that tests cover both membership and access card scenarios, adding one more test to our growing suite.

The problem with solely using code coverage metrics to measure the quality of automated tests is that none of the metrics evaluate whether my tests actually check whether the customer is allowed access, or how much the software calculates as a charge. The verification of these states and variables are not included in the metrics.

Measuring code coverage on your automated tests is great, but it is only part of the picture. Code coverage only tells you the logic and branches which have been executed, it doesn't really measure whether your tests are getting a lot of data of functional coverage, and it doesn't tell you whether your tests are effectively detecting failures.

Validation of the system response (effectively comparing actual to expected results) is a critical part of implementing automated testing, it is straightforward to check that a variable is sensible; however, as interfaces get more complex (think of an XML message or a user interface) the amount of design subjectivity around the validations increases.

Mutation operation engines, which apply mutation operators to code, vary in the operators they support, and indeed the user can usually configure how these are applied. As an illustration, the well-known Mothra study [4] and supporting tool used the operators on Fortran shown in Table 1. These are somewhat outdated, as operators have evolved with the development of object-orientated techniques [5]. Of 
Table 1 Mothra operators

\begin{tabular}{l|l}
\hline Type & Description \\
\hline aar & Array for array replacement \\
\hline abs & Absolute value insertion \\
\hline acr & Array constant replacement \\
\hline aor & Arithmetic operator replacement \\
\hline asr & Array for variable replacement \\
\hline car & Constant for array replacement \\
\hline cnr & Comparable array replacement \\
\hline csr & Constant for scalar replacement \\
\hline der & DO statement end replacement \\
\hline lcr & Logical connector replacement \\
\hline ror & Relational operator replacement \\
\hline sar & Scalar for array replacement \\
\hline scr & Scalar for constant replacement \\
\hline svr & Scalar variable replacement \\
\hline crp & Constant replacement \\
\hline dsa & Data statement alterations \\
\hline glr & Goto label replacement \\
\hline rsr & Return statement replacement \\
\hline san & Statement analysis \\
\hline sdl & Statement deletion \\
\hline src & Source constant replacement \\
\hline uoi & Unary operation insertion \\
\hline &
\end{tabular}

course, the volume of potential operators and their resulting mutations of software code is enormous, and no implementation can be viewed as exhaustive.

Running the software under test through a mutation operator could result in a number of changes to the code. Four example, rule-based operators are applied to this code:

\section{Pseudo Code}

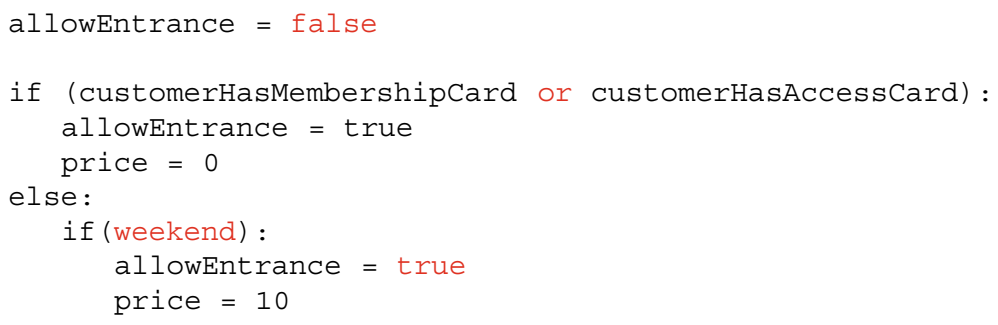


In this example, the red items will be changed. The first will be changed to be initialized as true, the second will change an or to an and, the third and fourth will negate a Boolean value by adding a not.

As a result, four versions of the system under test will be compiled, and the mutation testing routine should run all automated tests against each version. In order to successfully detect each of the mutants, the test suite would need the following characteristics:

- To test that the customer was not permitted access if the customer did not have either card, and it was not a weekend. This would test a failure to initialize the variable in mutation 1 .

- To test where a customer has one card, but not the other (full condition coverage would also require this), and then validate the customer was permitted access.

- To test that a customer was allowed access without cards on a weekend.

- Replacing variable values

As you can see, although a test suite built to achieve code coverage would exercise similar paths through the code, mutation testing metrics allow much more specificity about the verification the tests should perform.

This is useful because, unsurprisingly, many software faults are introduced in the coding process. For example, the common off-by-one error, where a programmer instructs a loop to iterate one time to many, or too few-or miscalculates a boundary condition-is directly addressed by test design techniques such as boundary value analysis and equivalence partitioning. Similarly, this kind of error is commonly injected by mutation test operators.

The mutation testing process can be summarized as follows:

1. First, mutants are created, by inserting errors.

2. After they have been created, the tests are selected and executed.

3. The mutants will be "killed" if the tests fail when executed against the mutant.

4. If the result of testing the mutant is the same as with the base software, the mutant has "survived."

5. New tests can be added, existing tests amended, or code refactored-in order to increase the number of "killed" mutants. Some mutations cannot ever be detected, as they produce equivalent output to the original software under test, these are called "equivalent mutants."

Once the full process has been executed, a mutation score can be calculated. This is the ratio of killed mutants, to the total number of mutants. The closer this score is to 1 , the higher the quality of the testing suite and the software.

$$
\text { Mutation Score }=\frac{\text { Number of Killed Mutants }}{\text { Total Mutants }}
$$




\section{Mutation Testing Challenges and Strategies}

Mutation testing is inherently scalable as it is usually based on doing something you already do automatically, many times over and independently. That said, this should also be true of automated integration and system testing, but is often not.

The resources required to conduct mutation testing can be significant, clogging up the continuous integration pipeline for hours to run endless iterations of tests on endless iterations of mutated software. Additionally the time required to go through and fix all the issues uncovered, can be daunting.

Three distinct areas of cost need to be considered:

- The compile-time cost of generating mutants

- The run-time cost of running tests on the mutants

- The human cost of analyzing results

\section{Compile Time}

As mentioned previously, a major problem with mutation testing is the cost of execution. The number of mutants is a product of the number of lines of code and the number of data objects, but as a rule of thumb the number of generated mutants is typically in the order of the square of the number of lines of code. Some strategies [6] have been tried to reduce the amount of execution:

- Sampling-Executing only a random sample of mutants across a logical area of software and associated tests.

- Clustering-Using unsupervised machine learning algorithms (e.g., K-means) to select mutants.

- Selective Testing-Reducing the number of mutation operators, that is, the heuristics used to inject faults, can reduce the number of mutants by 60

- Higher-order Mutation-First-order mutants are those that have had a single fault injection; second-order mutants have been injected with multiple faults, in multiple iterations of mutation. Higher-order mutants are harder to kill, and focusing solely on second-order mutants has been shown to lead to reduced effort, without reducing coverage.

- Incremental Mutation-Mutating only new or changed code, rather than the whole code base under test.

We know that defects cluster in the same areas of code, and perhaps a simple strategy of applying the techniques to limited, complex, high-risk, and defect-ridden areas of functionality can offer an appropriate balance of cost vs benefit.

Conversely, there are statements in code that we not be concerned about. For example, excluding all logging statements from mutation may be appropriate and lead to less mutants to test.

Another approach used to reduce the amount of time and resources required is direct integration with the compiler. Early mutation testing approaches compiled each mutant individually; however, more modern approaches compile once, and then mutate an intermediary form such as bytecode. This has significant benefits in 
terms of compilation performance, but no benefits in terms of the execution time of evaluating each mutant.

Much research has been conducted into one concept called "Weak Mutation." This effectively evaluates mutants at a component level, rather than fully executing each mutant. This has been found to be nearly as effective as "Strong" mutation with reduced runtime cost [6].

\section{Runtime}

The runtime cost of running mutation testing cycles can be significant. Of course, the execution can be scaled horizontally in most cases, as tests can be run across multiple machines, or it can be scaled vertically by using more powerful machines. To apply horizontal scalability it is necessary to consider this when selecting the appropriate mutation testing tools, with support for such approaches.

Classical test automation techniques for improving the run-time of tests also need to be considered. Hard-coded waits built into tests may not be noticeable when the test is run once, but scaled to hundreds or thousands of executions, become a significant cost. Optimization of automated tests for performance purposes should be performed before attempting to introduce mutation testing.

\section{Analysis}

Two related areas of challenge are the Oracle Problem, and the problem of reducing equivalent mutants. The Oracle Problem is far from unique to mutation testing, and applies to any area of testing where it is difficult to ascertain whether a test has succeeded.

This can occur when mutants can't be killed, because the assertions or validations required to be implemented in the tests are too difficult to implement.

It can also occur when software is less deterministic, and when it is difficult to understand whether the failure to detect a mutation is actually meaningful. I asked Alex Denisov, author of a mutation testing tool called Mull [7], what he thought the biggest challenges were:

As a user, the biggest issue so far is the test oracle problem. It is not a big deal on small or medium projects. However, for a big project, mutation can produce thousands of mutants, hundreds of which are surviving. It is not yet clear how a developer is supposed to process them—-manually reviewing those hundreds is simply impractical.-Alex Denisov

The problem of reducing equivalent mutants is also relevant, that is, the mutation does not result in observable change to the output or execution. This can occur because of dead code that isn't used; the mutation only changes the speed of the software; or, the mutation only changes internally used data that does not affect the end state or output. 
I asked Markus how he optimizes mutation testing to reduce equivalent mutants, and he disagreed with the metric:

This cannot be answered as is, the reality is more difficult. The question implies that equivalent mutations are a real world problem, but they are not. The question also implies the tool produces mutations of "low informational value" and not every mutation that comes back alive contains value for the human who reads a report. Both assumptions are false in my world. Equivalent mutants almost never show up, and low value mutations do not exist, hence everything must be killed.-Markus Schirp

To understand what Markus meant, let's look at an equivalent mutation:

\section{Pseudo Code}

def foo(i):

return $i+1+0$

An example of a potentially equivalent mutant would be to remove the "+ 0 ". While this would change the software under testing, it doesn't change the output, as adding zero to a number has no actual effect anyway. That is exactly the point, the code is irrelevant and should be removed.

\section{Mutation Testing Tools}

Mutation testing tools are numerous in nature, this is in part, because they are intrinsically linked with the programming languages used in the implementation. You can't generally use a tool designed for C++ with Java. It is crucial to pick a tool that supports the underlying technology stack, has support to allow you to configure the mutation heuristics you want, integrates with your development environment well, and supports parallel and distributed execution. Wikipedia [8] has a good list of mutation engines that you can use with your code. Getting started with these tools can be as simple as injecting a dependency into your build configuration.

Selecting and evaluating tools that suit you is actually more important than with traditional tool selection. One reason is that the tools will come with different default operators and strategies built in. As outlined earlier, these operators and strategies effectively dictate the technical approach and directly affect the results. The tool also may determine how you can scale the run-time execution, and choosing a tool with such limited support may result in impractical execution timelines.

Tools and libraries also exist for mutating data structures rather than code, for instance, applying heuristic operators against standard data structures such as XML. 
However it isn't just the core mutation engine that you need to consider:

As an implementor, the bigger problem I see is the smooth integration. How to include the mutation testing into existing infrastructure: various build systems, test frameworks, CI pipelines, IDEs, etc.-Alex Denisov

There are other types of tools that you might want to look at with regard to your overall workflow. For instance, Pitest, a Java mutation engine, has a Cucumber plugin [9] that allows you to integrate with Cucumber and Behavior-Driven Development. Similarly, SonarQube [10], the popular code quality monitoring suite, has plugins that allow you to display detailed results from your mutation test run. Some mutation testing engines also offer IDE plugins that accelerate the feedback loop and allow you to see the results in the context of your code.

\section{Other Applications of Mutation Testing}

One way to look at scaling mutation testing is in the test level. It is far easier to apply mutation testing at a unit test level, because individual mutations can be intuitively tracked to effects in the software's operation, less resources are required, and the start-up and execution time for each test is much smaller, leading to faster results.

Mutation testing can still be applied at higher test levels such as functional system testing. Mutated software versions can be deployed to web servers, and typical tools like Selenium can be used to run the test iterations. However the execution time, and amount of setup work, will be considerably higher. Investigating and resolving issues can also require more work to link the mutated line of code back to a functional test verification.

The concept of mutation testing has been proposed to cover other areas of software quality as well. For example, it has been proposed, at least in research [11], that mutation can be used on top of formal specification languages to detect defects at the design stage.

Mutation testing can also be used to inject faults in the running environment. Chaos Monkey, a tool originally developed by Netflix [12] to test the resilience of their environments, randomly injects infrastructure faults, to see how the application under test (or live application!) handles the failure. This can be viewed as mutating the environment the software runs in:

Failures happen, and they inevitably happen when least desired. If your application can't tolerate a system failure would you rather find out by being paged at $3 \mathrm{am}$ or after you are in the office having already had your morning coffee?-Chaos Monkey on github 
Fuzz testing, which mutates the input domain of an application, can also be considered a type of mutation testing. Instead of mutating the program code, it mutates inputs to the system under test. This has completely different goals, though, to the mutation testing described above. It is primarily aimed at detecting how your system will behave with unexpected inputs; this might be carried out as part of a security testing activity, or a negative functional test.

Finally, mutation testing can be used to understand the properties of an unknown code base:

mutation generation, without running the kill phase, allows a nice and unbiased detection of complex code structures.-Markus Schirp

This approach can be used to prioritize regression testing, or refactoring of a large code base.

While mutation testing has been around for a while, it is solidly building support in the engineering community, and it clearly delivers useful information it just isn't possible to get elsewhere.

\section{Conclusion}

This chapter has hopefully opened your eyes to a completely different approach to looking at test coverage. While mutation testing is something that is currently applied most frequently at the unit testing level, the concepts can be applied, and the benefits realized throughout a full set of software quality assurance practices.

It is not only an effective way to assess your automated tests, but also a way to understand the complexity of your code, and quantitatively understand your code quality and test coverage.

The concepts can also be applied to code, input data, the environment, and no doubt other technical artifacts.

As explained, the biggest challenges are the compute resources required to execute a large number of tests on a large number of mutants, and also the human resources required to analyze equivalent mutants, and solve oracle problems. Finding the right balance between coverage confidence and resource requirements is crucial. Limited research exists, which covers the financial and quality benefits and costs of mutation testing outside of research, and this is clearly an area that needs more analysis.

Maybe it can't help you refactor all your code or improve all your tests, but it can definitely point you in the right direction. No software quality assurance specialist needs less information, and no test suite can ever be fully understood in terms of coverage without some execution results. Mutation testing is no silver bullet, but cannot be ignored by true quality professionals. 
Acknowledgements I would like to thank the following people their help: Markus Schirp for his advice and quotes as author of the mutation testing tool Mutant; and, Alex Denisov from lowlevelbits.org, for his quotes as a user of mutation testing and author of the tool Mull; and, My better half Julia for letting me disappear into writing mode on weekends;

\section{References}

1. Mutant: Mutation testing for Ruby https://github.com/mbj/mutant

2. Lipton, R.: Fault Diagnosis of Computer Programs, student report, Carnegie Mellon University (1971)

3. Saxena, R., Singhal, A.: A critical review of mutation testing technique and hurdles, in 2017 International Conference on Computing, Communication and Automation (ICCCA), Greater Noida, pp. 887-892 (2017)

4. DeMillo, R.A., Guindi, D.S., McCracken, W.M., Offutt, A.J., King, K.N.: An extended overview of the mothra software testing environment. In: IEEE Proceedings of Second Workshop on Software Testing, Verification, and Analysis, July 1988, pp. 142-151

5. Kim, S., Clark, J.A., McDermid, J.A.: Class mutation: mutation testing for object-oriented programs, p. 15

6. Jia, Y., Harman, M.: An analysis and survey of the development of mutation testing. IEEE Trans. Softw. Eng. 37(5), 649-678 (2011)

7. Mull: Mull is a LLVM-based tool for Mutation Testing with a strong focus on C and C++ languages. https://github.com/mull-project/mull

8. Mutation Testing: Wikipedia. 03-Mar-2019. https://en.wikipedia.org/wiki/Mutation_testing

9. Pitest Cucumber Plugin: https://github.com/alexvictoor/pitest-cucumber-plugin

10. SonarQube: https://www.sonarqube.org

11. Sugeta, T., Maldonado, J.C., Wong, W.E.: Mutation testing applied to validate SDL specifications. In: Proceedings of the 16th IFIP International Conference on Testing of Communication Systems, Mar. 2004, p. 2741

12. Chaos Monkey github site: https://github.com/Netflix/SimianArmy/wiki/Chaos-Monkey

Open Access This chapter is licensed under the terms of the Creative Commons Attribution 4.0 International License (http://creativecommons.org/licenses/by/4.0/), which permits use, sharing, adaptation, distribution and reproduction in any medium or format, as long as you give appropriate credit to the original author(s) and the source, provide a link to the Creative Commons licence and indicate if changes were made.

The images or other third party material in this chapter are included in the chapter's Creative Commons licence, unless indicated otherwise in a credit line to the material. If material is not included in the chapter's Creative Commons licence and your intended use is not permitted by statutory regulation or exceeds the permitted use, you will need to obtain permission directly from the copyright holder. 